Revista Posgrado y Sociedad

Sistema de Estudios de Posgrado

Universidad Estatal a Distancia

ISSN 2215-2172

Costa Rica

revistaposgradoysoci@uned.ac.cr

\title{
Necesidad de unificar criterios de calidad para la mediación pedagógica de cursos en línea de la Universidad Estatal a Distancia
}

Need for unification of Pedagogical mediation's quality criteria in online courses at the Universidad Estatal a Distancia. Costa Rica.

\author{
Xinia Calvo C. $\quad$ Natalia Salas $Q$. \\ Programa de Apoyo Curricular y Evaluación de los \\ Aprendizajes, Universidad Estatal a Distancia.
}

Volumen 13, Número 1

Marzo 2013

pp. $27-44$

Recibido: Febrero, 2011

Aprobado: Julio, 2012 


\section{Resumen}

En los últimos años la educación superior ha experimentado entre sus más fuertes tendencias; la incorporación de las TIC en los procesos de formación. En atención a este interés, en el III Congreso Universitario de la Universidad Estatal a Distancia (2006) se aprobó como moción la incorporación progresiva del aprendizaje en línea en todos los planes de estudio; situación que ha generado un aumento significativo de cursos que hacen uso de las plataformas de aprendizaje en línea. A lo anterior, se agrega una serie de esfuerzos tendientes al mejoramiento del diseño e implementación de los cursos en línea; incluyendo el más reciente que se plasma el Plan de Desarrollo 2011-2015.

Si bien es cierto la institución requiere de recursos tecnológicos para hacer frente a la incursión de las TIC en los procesos de aprendizaje, esta tarea demanda además centrar el interés en la adecuada planificación y estructuración de los contenidos del curso; en aras de que la metodología y la integración de recursos se orienten al logro de los objetivos de aprendizaje. Al respecto, es importante destacar que en la Universidad Estatal a Distancia, cuenta a la fecha con un número significativo de cursos en línea, sin que en ellos medien criterios de calidad unificados que orienten la mediación pedagógica de los mismos.

Así las cosas, el propósito de este escrito es plantear la necesidad de establecer los criterios de calidad que se debe considerar en la mediación pedagógica de cursos en línea de la UNED- Costa Rica.

Palabras clave: mediación pedagógica; aprendizaje en línea; criterios de calidad 


\begin{abstract}
In the last years, higher education has experienced among its strongest tendencies; the incorporation of ICT in educational processes. In response to this interest, in the III University Congress of the Universidad Estatal a Distancia (2006) was adopted as a motion the progressive incorporation of online learning in all curricula; situation that has generated a significant increase in courses that make use of learning management systems. Foregoing, it adds a series of efforts aimed at improving the design and implementation of on-line courses; including the latest which translates the Development Plan 2011-2015.

Although the institution requires technological resources to deal with the incursion of the ICT in learning processes, this task requires further focus the interest in the proper planning and structuring the contents of the course; in order that the methodology and the integration of resources should be geared to the achievement of learning objectives. In this regard, it's important to point out, that at the time, in the Universidad Estatal a Distancia there are a significant number of online courses, without unified quality criteria to guide teaching mediation of them.

So, the purpose of this writing is raise the need to establish quality criteria which should be considered in the pedagogic mediation of on-line courses in the Universidad Estatal a Distancia-Costa Rica.
\end{abstract}

Keywords: pedagogical mediation; e-learning; quality criteria 


\section{Introducción}

La intención del presente artículo es plasmar la necesidad de unificar los criterios de calidad que deben privar en la mediación pedagógica de los cursos en línea que se ofertan en la Universidad Estatal a Distancia (UNED), en aras de generar una reflexión sobre la importancia de establecer líneas institucionales en torno al tema.

Con respecto a los objetivos del artículo se propone un objetivo general y algunos específicos:

Objetivo general:

- Plantear la necesidad de establecer criterios de calidad para la mediación pedagógica de cursos en línea de la Universidad Estatal a Distancia.

Objetivos específicos:

- Bosquejar un referente teórico básico respecto a la temática de la mediación pedagógica de los cursos en línea.

- Conocer los principales esfuerzos institucionales tendientes a la incorporación e implementación de los cursos en línea, como respuesta a una de las megas tendencias en educación superior.

- Determinar criterios que son considerados por algunas personas encargadas del diseño curricular de cursos, para establecer la mediación pedagógica de los cursos en línea de la UNED

\section{Breve referente teórico acerca de la temática}

Tendencias de la Educación Superior en el uso de las TIC

La época actual se caracteriza por la existencia de variadas formas de comunicación y por la gran cantidad de información con que se puede contar sobre los diversos temas o situaciones que suceden en el contexto mundial; lo cual es posibilitado por las diferentes herramientas tecnológicas desarrolladas por el ser humano en las últimas décadas con una evolución y mejoramiento constantes por empresas dedicadas al desarrollo de tecnologías de comunicación e información (TIC).

Este desarrollo tecnológico ha propiciado que alguna parte de la población estudiantil que ingresa a las universidades, tenga un mayor acceso a documentos y a datos, interactúen con personas en forma asincrónica sin importar las barreras geográficas y de idiomas y además se vinculen a diferentes redes sociales que les permiten interactuar en espacios donde pueden colocar imágenes, textos y sonidos.

De esta manera, el contexto social actual hace que el proceso de formación de profesionales requiera de un cambio sustancial en la manera en que se planifica, se implementa y se evalúa. Al respecto, (Gazzola y Didriksson, 2008) señala que las universidades tienen como principal reto poder asumir, de manera reflexiva y crítica, la perspectiva de la sociedad del conocimiento, donde se debe diseñar y poner en práctica "una oferta que pueda ofrecer la más amplia gama de experiencias científicas, tecnológicas y humanísticas que hagan posible un salto de calidad en la responsabilidad social y el compromiso de las instituciones de educación superior en la región". (p.32) Esto significa, el desarrollo de capacidades para la resolución de problemas, para 
trabajar de manera colaborativa, para investigar, entre otras.

Ante tal situación, en la Conferencia Mundial de Educación Superior de 1998 se establece como una de las misiones y funciones de la educación superior la incorporación de las TIC en el proceso formativo, esto con el fin de propiciar mejoras en su calidad y dar un mayor acceso a la población, de manera que se aproveche el potencial y los desafíos que plantea el uso de las TIC. Desde entonces, las universidades han ido incursionado poco a poco en la incorporación de las TIC en sus procesos educativos.

Aunado a lo anterior, la educación superior en América Latina se enfrenta actualmente a un cambio en un su estructura donde se pasa de un campus físico (en el caso de las universidades con sistema presencial) a macrouniversidades con espacios de aprendizaje en la virtualidad. En el caso de las universidades con modalidad a distancia se pasa del uso de medios impresos al uso de medios virtuales, multimediales y las tecnologías móviles. Asimismo, existe una creciente importancia a los procesos de internacionalización de la oferta académica de las universidades, según Gazzola y Didriksson (2008) mediante redes académicas, la movilidad de estudiantes y profesores, así como nuevos procesos de transferencias de conocimientos y tecnologías.

$\mathrm{Al}$ respecto, Seoane y García (s.f.) han identificado cuatro generaciones del e-learning. La primera consiste en la implementación de la infraestructura tecnológica, el desarrollo de herramientas de comunicación y el traslado del proceso formativo al soporte en línea. La segunda se distingue por la necesidad de la calidad en el e-learning así como la propuesta de un modelo de formación con apoyo de las TIC, acompañada por un proceso de capacitación de su personal docente y administrativo. La tercera en el caso de la UNED, se podría decir que su oferta educativa se encuentra en un proceso de transición a la segunda generación, pues si bien es cierto existen carreras que están empezando a incursionar en la virtualización, hay otras que tienen un alto grado de virtualidad en los cursos que oferta y que en el análisis de su implementación han visto la necesidad de realizar mejoras que les permitan procesos educativos cada vez de mayor calidad.

Esta preocupación por la calidad de los cursos en línea, se manifiesta también desde la Conferencia Mundial de Educación Superior realizada en el año 2009, en la cual ratifica lo estipulado diez años antes en esa misma conferencia y añade la necesidad de contar con políticas e infraestructuras que permitan aprovechar el potencial de las TIC.

En este sentido, Vessuri, en compilación de Gazzola y Didriksson, 2008, menciona que es necesario que los sistemas formativos se planteen de manera que orienten a una construcción de conocimientos desde el aprender a aprender, por medio de 
uso eficaz de las herramientas tecnológicas y estrategias pedagógicas innovadoras y propulsoras de aprendizajes complejos, centrados en el estudiante y no en el profesor como poseedor de todo conocimiento.

Ollio (2009) citado en UNESCO 2009a lo cual significa una modificación importante en la metodología utilizada en la educación superior, en la que el profesorado asume cada vez más un papel de mediador y el estudiantado adquiere una mayor autonomía y regulación de su propio aprendizaje.

La mediación pedagógica en línea

Se puede entender la mediación pedagógica como el proceso en el cual se facilita una serie de herramientas y estrategias didácticas mediantes las cuales se favorece un enlace entre el estudiantado y el objeto de conocimiento. Este proceso de mediación tiene como punto de partida las experiencias previas de cada estudiante, sus necesidades y características, desde las cuales se establece su zona de desarrollo real para tener claridad acerca de las áreas que se requiere fortalecer y mejorar para la optimización de su proceso de construcción de conocimientos. (DíazBarriga y Hernández, 2004; Bautista et al, 2008; Barberà et al, 2011; Barberà, 2006)

En el caso del aprendizaje en línea, este proceso de mediación pedagógica se lleva a cabo por medio de las TIC, cuyo aspecto central es el proceso de comunicación e interacción, entre estudiantes- profesorado y entre pares; pues a través de éste se hace posible la construcción conjunta de los aprendizajes.
Según Salmon (2004, citado por UNED, 2011) el desarrollo de un proceso de aprendizaje en línea conlleva 5 momentos que contemplan tanto los requerimientos tecnológicos como los de interacción entre las personas participantes. Valga aclarar Estos momentos son:

- Acceso y motivación: Se brinda inducción al estudiantado sobre el uso de la plataforma de aprendizaje en línea, se realizan actividades de motivación inicial, se realiza el diagnóstico de conocimientos previos de cada estudiante y se explora la organización del curso en el entorno virtual.

- Socialización: Se abren espacios de comunicación social en los que se propicia la interacción entre las personas participantes y el profesorado y se implementan las normas de comunicación.

- Intercambio de información: Se desarrollan estrategias para la autonomía del estudiantado, se facilitan recursos para favorecer la interacción con los contenidos del curso y su profundización.

- Construcción de conocimientos: Se desarrollan actividades que permitan la construcción de conocimientos por medio de la colaboración, el pensamiento crítico, la creatividad y el pensamiento práctico. Asimismo, se crean espacios para el debate de ideas, solución colaborativa de problemas, proyectos, entre otros.

- Desarrollo: Se propicia la metacognición para el control y comprensión de los procesos de pensamiento, se desarrollan 
actividades donde se aplique lo aprendido, en diferentes contextos y situaciones; además se abren espacios para la reflexión.

En cada uno de estos momentos el proceso de mediación pedagógica tiene diferentes finalidades, mientras que en el primero requiere de una mediación que promueva la participación y motivación del estudiantado, en los tres últimos se requiere de un proceso donde se favorezca el pensamiento crítico, reflexivo, la metacognición y la coconstrucción de conocimientos, mediante la propuesta de experiencias de aprendizaje, capaces de generar aprendizajes significativos y que propicien desarrollo de altos niveles de conocimiento.

En este sentido, la mediación pedagógica en los entornos virtuales, según Cabero y Román (2008) tiene ocho variables críticas que garantizan el éxito de la acción formativa, las cuales se describen a continuación:

- estrategias didácticas: es necesario propiciar la actividad individual y el trabajo colaborativo, por lo que es importante que el profesorado tenga un dominio de diversas estrategias.

- papel del profesorado: se caracteriza por ser un diseñador de situaciones mediadas de aprendizaje.

- papel del estudiantado: debe poseer independencia, motivación y autosuficiencia.
- Contenidos: deben ser relevantes, acordes con los objetivos de aprendizaje, sin sobre cargar la asignatura y se deben estructurar de acuerdo a su nivel de complejidad aprovechando la interactividad, hipertextualidad y multimedios que ofrece las TIC.

- herramientas de comunicación: requieren ser preparadas con anterioridad por el profesor, deben permitir la comunicación por diferentes vías y de forma sincrónica así como asincrónica.

- modelos de evaluación: deben ser congruente con la metodología utilizada, a la vez que propicie la autorregulación y la metacognición, en el cual se tenga claridad acerca de qué se requiere evaluar, cuándo y para qué.

- aspectos organizativos: debe organizarse lo administrativo y lo académico como un sistema.

- e-actividad: debe ser pertinentes a los objetivos y contenidos de aprendizaje, debe facilitarse en un espacio de tiempo suficiente para ser realizada, deben ser variadas pero sin sobrecargar al estudiantado pues se puede caer fácilmente en el activismo sin propósito, deben favorecer la interacción entre las personas participantes; además deben ser acordes al nivel educativo en que se encuentra la asignatura.

Cada una de estas variables se encuentra interrelacionada y la manera en que se implementen va a 
definir la calidad del proceso de formación. Así por ejemplo, se podría contar con herramientas de comunicación de última tecnología, pero si no se sabe cuál es el momento propicio para utilizarlas o para qué tipo de proceso de aprendizaje es más apropiada, se convertirá en una herramienta poco útil para la construcción de los conocimientos lo que finalmente podría causar desinterés y confusión en el estudiantado.

Por tanto, se puede decir que para la mediación pedagógica es indispensable planificar previamente la acción formativa a partir del fundamento teórico y metodológico establecido desde el plan de estudios de la carrera, del diagnóstico de las características de la población a atender, de las herramientas con que cuenta la institución para la implementación de entornos virtuales de aprendizaje, así como del proceso reflexivo para orientar la construcción de conocimientos en el momento oportuno brindando la realimentación que sea necesaria.

El establecimiento de criterios de calidad para la mediación pedagógica de procesos de aprendizaje en línea

Una de las principales expectativas de las universidades al incorporar las TIC en los procesos de formación, es que dichos procesos mejoraran en cuanto a su calidad educativa, en tanto que su implementación permitiera responder a las características y exigencias de la actual sociedad del conocimiento; sin embargo, la experiencia ha dejado ver que usar las TIC no es suficiente, sino que tiene que ver con cómo se desarrolla el proceso de construcción de conocimientos.

Tal y como se mencionó anteriormente, Seoane y García (2006) sugieren que esta etapa en el aprendizaje en línea responde a una segunda generación determinada por la preocupación de contar con procesos formativos de calidad. De esta manera, ellos proponen la siguiente definición de calidad ...la efectiva adquisición de una serie de competencias, habilidades, conocimientos y destrezas por parte de un conjunto de alumnos, mediante el desarrollo de contenidos de aprendizaje adecuados, impartidos a través de unas herramientas web eficientes y con el apoyo de una red de servicios añadidos, cuyo proceso desde el desarrollo de los contenidos hasta la adquisición de las competencias y el análisis de la intervención formativa en su conjunto- está garantizado por un exhaustivo y personalizado proceso de evaluación y certificación, y monitorizado por un equipo humano que ejerce una labor tutorial integral durante todo el proceso de enseñanza-aprendizaje. (p. 2)

Como se puede deducir, la calidad es determinada no por el uso de TIC sino por la manera en que se desarrolla el proceso de aprendizaje, por tanto, se considera como fundamental la mediación que se lleve a cabo en dicho proceso. Desde esta misma línea, la Conferencia Mundial sobre Educación Superior del año 2009, señala que los criterios de calidad que se planteen deben tener la finalidad de propiciar el pensamiento crítico e 
independiente, el aprendizaje permanente, la innovación y la diversidad, lo cual responde al planteamiento metodológico en el que se basa el proyecto de formación.

Adicionalmente, Barberà, Mauri y Onrubia (2011) señalan tres motivos esenciales para evaluar la calidad del uso de las TIC en la educación: los educativos, los socioculturales y los tecnológicos (p. 21). Los motivos educativos se basan en la necesidad de contar con orientaciones claras sobre el análisis del proceso de formación que se lleva a cabo, el papel del profesorado y del estudiantado. Los motivos socioculturales se justifican desde la celeridad de los cambios en las tecnologías y la brecha digital existente entre distintas zonas del país, el aumento de ofertas educativas, así como la tendencia mundial de certificación de la calidad de la educación en sus distintas modalidades.

Por otra parte los motivos tecnológicos se refieren a la presencia cada vez más evidente de la tecnología en los diferentes campos de la vida y a tener acceso a la información, al igual que la forma en que se está llevando a cabo la transferencia de los recursos tecnológicos al campo educativo. En este sentido, a nivel mundial existen propuestas de instrumentos para evaluar la calidad de los entornos virtuales, algunas de ellas más centradas en lo que se refiere a componentes tecnológicos en cuanto a la accesibilidad y calidad de los recursos que se utilizan, mientras que otras abarcan lo referente a la planificación didáctica de los cursos. Un ejemplo de estas propuestas es la creación de la norma de gestión de calidad de la formación virtual elaborada por el Instituto de Normas Técnicas de Costa Rica (INTECO), la cual va dirigida sobre todo al ámbito de las capacitaciones o de educación no formal que utilizan las TIC, la cual contiene factores de satisfacción relacionados con aspectos tecnológicos y a lo que ellos han llamado la facilidad de asimilación, es decir la capacidad del soporte tecnológico para favorecer el proceso de aprendizaje de la persona usuaria.

Otro ejemplo es la Guía de Evaluación para Cursos Virtuales de Formación Continua desarrollada por el Instituto Latinoamericano y del Caribe de Calidad en Educación Superior a Distancia quien se encarga de certificar cursos virtuales. Esta guía contempla el área de tecnología, la de formación del profesorado y del estudiantado, el diseño instruccional (incluye las orientaciones para el curso, elementos curriculares, seguimiento y tutoría y evaluación) y el servicio de información y soporte que se brinda al estudiantado.

Por su parte Barberà y otros (2011), plantean una serie de instrumentos de evaluación de la calidad de la formación en línea a partir de la experiencia de su práctica educativa, estos instrumentos rescatan el aspecto tecnológico así como el pedagógico, indicando que si bien es cierto es importante contar la tecnología y que 
su funcionamiento sea de calidad, no se puede dejar de lado la propuesta pedagógica que se implementa en los entornos de aprendizaje en línea, esto debido a que el sustento pedagógico es fundamental para garantizar la calidad del proceso de formación del estudiantado.

Dentro de su propuesta, los autores incluyen varios instrumentos para valorar la calidad de los siguientes aspectos: diseño tecnopedagógico de los materiales educativos multimediales, el diseño tecnológico y pedagógico, el desarrollo del proceso formativo, además de instrumentos para valorar cada uno de los aspectos mencionados pero desde la perspectiva del profesorado y del estudiantado.

De esta manera, la UNED al tender desde su plan de desarrollo, al aprendizaje a través de las TIC, es necesario que los procesos de mediación pedagógica sean orientados a través de una serie de criterios de calidad, que permitan realizar procesos educativos acordes con los fines de la institución. Esto permitiría que las personas encargadas de cada carrera y de cada cátedra, tengan claridad acerca de lo que se espera en términos de procesos formativos; asimismo, las instancias asesoras y capacitadoras también tendrían un respaldo para poder orientar el proceso de formación del profesorado.

\section{Esfuerzos tendientes a la incorporación e implementación de los cursos en línea}

La Universidad Estatal a Distancia, en la última década y siguientes años, ha realizado esfuerzos en procura de incorporar e implementar los cursos en línea.

En un primer acercamiento, se destacan las mociones aprobadas en el II y III Congreso Universitario. En el II Congreso Universitario: Por un cambio con participación real, celebrado en el año 2000, las mociones 22 y 23, plasman la necesidad de considerar los medios que contribuyeran a mejorar el aprendizaje, además de las unidades de texto; así mismo se planteaba la necesidad de "Propiciar un desarrollo tecnológico acorde con las necesidades institucionales y sociales, tanto de índole académica como administrativa, y promover su uso racional y eficaz" ( Mociones II Congreso Universitario ).

Con respecto al III Congreso Universitario, "Por el fortalecimiento de una academia con calidad", celebrado en el año 2006, la moción 2, plasma la necesidad de "Establecer un plan de incorporación progresiva del aprendizaje en línea en todos los planes de estudio de la UNED, que considere diferentes escenarios, donde se tomen en cuenta los recursos educativos disponibles en la Institución en particular en los centros universitarios, la diversidad cultural de los estudiantes, la universalización de los medios 
empleados....." (Mociones III Congreso Universitario).

A partir de estas líneas, la universidad ha venido dando pasos, que han permitido la incorporación de las TIC, a los procesos de aprendizaje. Así entonces, a la fecha se identifican una serie de esfuerzos personales, y algunos colaborativos, que redundan en documentos institucionales, ponencias, artículos y trabajos finales de graduación para optar por un grado académico sobre temáticas como: perfil de competencias del docente en línea, desarrollo del pensamiento de orden superior en los cursos en línea, fundamentos y estándares de calidad en la educación en línea, estrategias metodológicas para el uso de herramientas de comunicación en los cursos en línea, entre otras, todas ellas afines a la mediación pedagógica de los curso en línea.

El documento: Cómo diseñar y ofertar cursos en línea. Consideraciones generales, es evidente muestra de lo anterior. Este expresa en su introducción que el mismo "reúne un conjunto de consideraciones que deben ser tomadas en cuenta cuando ofertamos cursos por medio de la red: el contexto educativo que se refiere a la creación de cursos en línea, el estudiante a quien va dirigido, los recursos institucionales; todo ello para favorecer nuevas estrategias de formación, que propicien el diseño de cursos en línea con criterios de calidad" (PAL-PACE-UNED, 2011) Aunado a lo anterior, como parte de las más recientes intenciones se cita el Plan de Desarrollo Institucional (2011-2015) que puntualiza en el área estratégica: Pertinencia y calidad, el objetivo estratégico, que indica la necesidad de "Renovar el modelo educativo de la universidad, permitiendo la introducción de nuevas tecnologías, innovaciones metodológicas y pedagógicas (p. 46). Respecto al incremento de cursos en línea, el Programa de Aprendizaje en línea (PAL), reporta que en el año 2001 se ofrecieron 45 cursos con componentes parcial o totalmente virtuales (citado por Salas, 2011), mientras que el informe de labores del 2010, de este mismo programa reporta la siguiente situación en torno a los cursos por plataforma tecnológica. 
Cuadro 1. Informe estadísticas de cursos por plataforma tecnológica. Cursos en línea en Microcampus, WebCT, Blackboard Learn y Moodle

\begin{tabular}{|l|l|l|l|l|l|l|}
\hline \multicolumn{1}{|c|}{ Años } & 2006 & 2007 & $\mathbf{2 0 0 8}$ & $\mathbf{2 0 0 9}$ & $\mathbf{2 0 1 0}$ & Totales \\
\cline { 1 - 6 } Microcampus & & & & & & \\
\hline WebCT & 391 & 304 & 157 & 56 & --- & 908 \\
\hline Blackboard Learn & --- & 136 & 354 & 465 & 536 & 1491 \\
\hline Moodle & --- & ---- & ---- & ---- & 54 & 54 \\
\hline TOTALES & --- & --- & 18 & 103 & 181 & 302 \\
\hline
\end{tabular}

Fuente: Informe de labores. PAL (2010).

La demanda de cursos en línea, ha redundado en la necesidad de asesoría curricular para el diseño de estos cursos; siendo esta experiencia, la principal fuente de la situación planteada en el presente artículo. Sobre este particular, debe acotarse que la práctica de las autoras como asesoras curriculares del Programa de Apoyo Curricular y Evaluación de los Aprendizajes (PACE), respalda la necesidad de establecer criterios de calidad en los cursos en línea que se oferten. A la fecha, con el mejor deseo de responder a las necesidades institucionales y sociales, se brinda toda una gama de cursos, sin criterios homologados que permitan asegurar un nivel de calidad óptimo desde el punto de vista pedagógico. Así entonces, los responsables del diseño de curso asumen los criterios que consideran y sobre estos realizan la propuesta curricular de curso. En este aspecto hay que destacar el hecho que la persona asesora curricular tiene poca injerencia en la elección de los criterios que se asumen para la mediación pedagógica; puesto que el rol de asesoría no es vinculante en las decisiones. A lo anterior, debe agregarse que en algunas ocasiones la persona o equipo responsable del diseño del curso cuenta con poca o ninguna experiencia en la educación en línea, sin embargo se le ha dado carga laboral para el diseño.

Es importante destacar que si bien es cierto la institución ha venido trabajando en los últimos años con mayor demanda de cursos en línea, institucionalmente no se cuenta con criterios de calidad establecidos; pero si existe una serie de esfuerzos que tienden a ofrecer un marco de acción en torno al tema a la calidad de los cursos en esta modalidad.

Finalmente, el IV Congreso Universitario, establece la necesidad de someter a revisión la práctica 
educativa de la UNED, con el fin de determinar cambios que permitan actualizar el modelo de gestión institucional. Desde este punto de vista, el presente artículo responde a los fines que se persiguen, por cuanto pretende tomar la práctica del aprendizaje en línea y ofrecerle opciones de mejoramiento, en cuanto a la calidad se refiere.

\section{La mediación pedagógica de cursos en línea de la UNED. Opinión de algunos encargados del diseño del curso}

Se realizó un trabajo exploratorio para conocer cuáles son los criterios de calidad que utilizan algunas personas responsables del diseño e implementación de los cursos en línea de la UNED.

Con respecto al trabajo realizado con algunos encargados del diseño del curso, el mismo se concretó con una entrevista. La anterior se realizó a algunas personas encargadas de cátedra o profesores de la universidad con experiencia en el diseño e implementación de cursos en línea de las diferentes escuelas y posgrado. Para recopilar la información de los encargados de cátedra y profesores se elaboró una entrevista con ocho preguntas abiertas que se enviaron por medio del correo electrónico a un total de 25 personas de las diferentes instancias académicas. Sin embargo, solamente contestaron siete personas, de las cuales tres son de la Escuela de Ciencias de la Educación, una de la Escuela de Ciencias de la Administración, una de la Escuela de Ciencias Exactas y Naturales, una de la Escuela de Ciencias Sociales y Humanidades y una del Sistema de Estudios de Posgrado. Para el análisis de la información recopilada se establecieron 8 categorías agrupadas en dos grandes dimensiones, las cuales se presentan en el siguiente cuadro. 
Cuadro 2. Categorías de análisis de información

\begin{tabular}{|c|c|}
\hline $\begin{array}{l}\text { 1. Diseño y organización del } \\
\text { proceso de aprendizaje }\end{array}$ & $\begin{array}{l}\text { 2. Desarrollo de la mediación } \\
\text { pedagógica (interacción) }\end{array}$ \\
\hline $\begin{array}{l}\text { Momentos del proceso formativo: } \\
\text { Se refiere a cada una de las etapas de } \\
\text { formación que se deben realizar para } \\
\text { la construcción del aprendizaje en } \\
\text { ambientes virtuales. Estos } \\
\text { momentos, según Salmon (2004), } \\
\text { son: Acceso y motivación, } \\
\text { Socialización, Intercambio de } \\
\text { información, Construcción de } \\
\text { conocimientos, Desarrollo. }\end{array}$ & $\begin{array}{l}\text { Conocimientos previos: Conjunto } \\
\text { de saberes que cada estudiante ha } \\
\text { construido a partir de experiencias de } \\
\text { aprendizaje formal y no formal, previo } \\
\text { al ingreso a un curso o espacio de } \\
\text { formación. }\end{array}$ \\
\hline $\begin{array}{l}\text { Número de actividades: Cantidad } \\
\text { de actividades que se planifican y se } \\
\text { ponen en práctica dentro de un curso } \\
\text { en línea. }\end{array}$ & $\begin{array}{l}\text { Actividades que favorezcan el } \\
\text { trabajo colaborativo: Conjunto de } \\
\text { actividades propuestas en un curso } \\
\text { que permiten el desarrollo de } \\
\text { habilidades de colaboración y de } \\
\text { comunicación así como la interacción } \\
\text { entre un grupo de estudiantes para el } \\
\text { logro de una meta común a partir de } \\
\text { un esfuerzo coordinado. }\end{array}$ \\
\hline $\begin{array}{l}\text { Duración de las actividades: Se } \\
\text { refiere al tiempo que se estima para } \\
\text { el desarrollo de una o varias } \\
\text { actividades dentro de un curso en } \\
\text { línea. }\end{array}$ & $\begin{array}{l}\text { Actividades que favorezcan el } \\
\text { pensamiento crítico: Conjunto de } \\
\text { actividades propuestas es un curso } \\
\text { que favorecen la reflexión, análisis y } \\
\text { valoración de situaciones diversas } \\
\text { para la resolución de problemas y } \\
\text { toma de decisiones de manera } \\
\text { fundamentada y crítica. }\end{array}$ \\
\hline $\begin{array}{l}\text { Elección de las herramientas de } \\
\text { aprendizaje en línea: selección de } \\
\text { la cantidad y diversidad de } \\
\text { herramientas que favorecen el } \\
\text { proceso de aprendizaje, acorde con } \\
\text { las necesidades, intereses y y } \\
\text { características de la población } \\
\text { atendida y de un curso en línea. }\end{array}$ & $\begin{array}{l}\text { Interacción entre pares y entre } \\
\text { profesor-estudiante: proceso de } \\
\text { intercambio de ideas, pensamientos } \\
\text { y reflexiones con otras personas sean } \\
\text { estas un profesor u otro estudiante, } \\
\text { para la construcción colaborativa de } \\
\text { conocimientos. }\end{array}$ \\
\hline
\end{tabular}

Fuente: Elaboración

Luego de recopilar los datos, se procedió a agrupar las respuestas de las personas entrevistadas según cada

una de las categorías mencionadas. A continuación se presentan los resultados. 


\section{Resultados}

De acuerdo con los datos recopilados de las entrevistas y a partir de las ocho categorías de análisis de información, se tuvo la siguiente información:

Respecto a los momentos del proceso formativo cuatro de las siete personas muestran desconocimiento acerca de los momentos formativos como tal, sin embargo, en la respuesta a la pregunta sobre cómo se favorece la interacción, dos personas mencionan foros de presentación los cuales corresponden al momento que Salmón (2006) denomina como de socialización. Una persona no responde a esta pregunta.

En cuanto al número de las actividades cada una de las personas entrevistadas manifestó criterios diversos, se coincide solamente en: complejidad de las temáticas del curso y el nivel académico al que pertenece. Otros criterios mencionados fueron: cantidad de objetivos a lograr, cantidad de tiempo que se extiende el curso, grado de virtualización, nivel de conocimiento del estudiante, tipo de conocimiento (experimental, conceptual, teórico-práctico), disciplina profesional, tipo de evaluación, número de estudiantes, afinidades a cierto tipo de actividades. Tres personas mencionaron cantidades de actividades de curso, que de acuerdo a su experiencia han determinado como idóneo, no obstante esas cantidades difieren.
Uno mencionan una actividad por unidad, otro que no más de ocho actividades por curso, y otro solo una actividad por semana.

En cuanto a la duración de las actividades solo dos personas hicieron referencia a este punto. Una de ellas, por su experiencia, indica que cada actividad debe durar mínimo 15 días. Otra señala que se establece un cronograma por módulo. $\mathrm{Si}$ bien es cierto, el número de actividades por curso en línea y la duración de cada una de ellas, podría diferir por la misma naturaleza del curso y por las características de la población a atender, es importante que las personas al planificar e implementar sus cursos, tengan claro cuáles son los criterios que son necesarios tomar en cuenta para brindar un curso de calidad, más allá de las experiencias particulares.

Respecto a la elección de las herramientas de aprendizaje en línea tampoco existe coincidencia en la mayoría de los criterios sugeridos. Aunque se repite en dos ocasiones los criterios: objetivos de aprendizaje del curso y temática a desarrollar.

Los demás criterios mencionados hacen referencia a las características de las herramientas en cuanto a facilidad de uso y accesibilidad, que permitan la mediación, que favorezcan la interacción, el grado de virtualidad, la modalidad en que se imparta, complejidad del tema, nivel académico en que se ubica el curso, tipo de conocimiento, tipo de evaluación, disciplina profesional, 
número de estudiantes, novedad de la herramienta, experimentación con una herramienta nueva.

Una persona indica que esa elección se realiza según criterio personal. Otra no responde.

En cuanto a conocimientos previos, la mayoría de personas entrevistadas indican que no han tomado en cuenta ese bagaje previo del estudiantado para el curso, incluso una de ella menciona que "los estudiantes no tienen conocimientos previos".

Los demás entrevistados señalan que mediante actividad de foros determinan esos conocimientos previos, al realizar preguntas generadoras, discusiones grupales y en actividades de aplicación de conocimientos. Una de las personas menciona que esto se hace porque se valoran los contenidos de cursos que lo anteceden, mientras que otra señala que por el tipo de curso, eso lo determina el tutor.

Respecto al tipo de actividades que favorezcan el trabajo colaborativo, cinco de las personas entrevistadas indica que sí las utiliza en sus cursos. Algunas de actividades que son mencionados mayormente son los foros de discusión, mapas conceptuales, el desarrollo de wikis, blogs grupales y WebQuest. Dentro de los beneficios que mencionan están la interacción y el desarrollo de habilidades de colaboración, así como el trabajo en equipo.

De las limitaciones de este tipo de actividades, las personas consultadas señalan la falta de habilidades para trabajar en equipo que tienen los estudiantes, así como que al estudiante o al profesor no le guste este tipo de actividad. Sin embargo, una de las personas considera que el trabajo colaborativo no tiene limitaciones.

Por otra parte, seis de las personas entrevistadas consideran que las actividades que favorecen el pensamiento creativo son los foros de discusión, sin embargo, resaltan que el pensamiento creativo se desarrolla principalmente por la actitud y la mediación que realice el profesor. Solo tres de las personas entrevistadas mencionaron beneficios de este tipo de actividades, dentro de los cuales consideran: el enlace entre teoría y práctica, la promoción de la autogestión y libertad de pensamiento, y que obligan al estudiante a leer y actuar críticamente al comentar el trabajo de los demás compañeros. En cuanto a las limitaciones, también solo tres personas responden indicando que la principal limitación es la cantidad de tiempo que se requiere para poner en práctica y evaluar este tipo de actividades.

\section{Conclusiones}

De acuerdo a la información recopilada y a la revisión bibliográfica realizada como marco del presente artículo, se concluye básicamente:

- La UNED ha incrementado y mantiene en aumento la incorporación de la virtualidad desde las diferentes propuestas de plan de estudios.

- La Universidad Estatal a Distancia, requiere de recursos tecnológicos para hacer frente a la 
incursión de las TIC en los procesos de aprendizaje, pero prioritariamente debe ocuparse de la calidad de los cursos en línea que se ofertan.

- El grupo de personas que participaron en la entrevista, muestran diferencias en cuanto a los criterios que utilizan para la mediación pedagógica de sus cursos. Sobre este particular, llama la atención el hecho de que se desconozca los momentos que debe tener el proceso formativo. Esto hace recomendable la realización de una investigación en la universidad que permita ahondar en aquellos criterios que se utilizan para la mediación pedagógica de los cursos en línea.

- Los responsables del diseño de un curso en línea asumen los criterios de calidad que consideren pertinentes para la mediación pedagógica, sin que la asesoría curricular tenga un marco unificado a nivel institucional.
- La mediación pedagógica de un curso en línea debe favorecer el proceso de comunicación e interacción, entre estudiantesprofesorado y entre pares, en procura de la construcción conjunta de los aprendizajes. En este sentido la UNED debe tener líneas homólogas que favorezcan los logros esperados

- Se requiere unificar a nivel institucional criterios de calidad para la mediación pedagógica de cursos en línea

- Se recomienda que a nivel institucional las instancias pertinentes y personas expertas en la temática coordinen para establecer los criterios de calidad para la mediación pedagógica de los cursos en línea y que esta sea socializada con los interesados para lo que corresponde.

\section{Referencias}

Barberà, E. (2006). Los fundamentos teóricos de la tutoría presencial y en línea: una perspectiva socioconstructivista. Estudios de Psicología y Ciencias de la Educación. Universitat Oberta de Catalunya. Educación en Red y Tutoría en Línea (págs.: 151:168). UNAM FES Zaragoza, México. Recuperado de http://www.tutoria.unam.mx/EUT2010/memoriaEUT/doc/Losfundamentosteoricosdelatutoriaenlinea. pdf

Barberà, E.; Mauri, T. y Onrubia, J. (coordinadores) (2011) Cómo valorar la calidad de la enseñanza basada en las TIC. Pautas e instrumentos de análisis. ( $3^{\circ}$ reimpresión). España: Grao.

Bautista, G; Borges, F. y Forés, A. (2008) Didáctica universitaria en entornos virtuales de enseñanzaaprendizaje. ( $1^{\circ}$ reimpresión) España: Narcea.

Cabero, J. y Román, P. (2008) E-actividades: un referente básico para la formación en Internet. ( $1^{\circ}$ reimp.) Sevilla: Eduforma.

Díaz-Barriga, F. y Hernández, G. (2002) Estrategias docentes para un aprendizaje significativo, una interpretación constructivista. Segunda Edición. México: McGraw-Hill. 


\section{Xinia Calvo \& Natalia Salas}

Gazzola, A. y Didriksson, A. (Eds.) (2008) Tendencias de la educación superior en América Latina y el Caribe. Bogotá: IELSAC-UNESCO. Recuperado de http://www.iesalc.unesco.org.ve/index.php?view=article\&catid=3\%3Acontenido\&id=2\%3Atendencias\& format=pdf\&option $=$ com content\&Itemid $=408 \&$ lang=es

Instituto Latinoamericano y del Caribe de Calidad en Educación Superior a Distancia. (2009) Guía de evaluación para cursos virtuales de formación continua. Loja: Universidad Técnica Particular de Loja.

Mociones de Congresos (2011) Recuperado de www. http://www.uned.ac.cr/ivcongreso/ .

Salas, I. (2011). El Efecto del Rol Docente Sobre el Pensamiento Crítico de los Foros en Línea en Educación Superior. Disertación Aplicada Presentada al Fischler School of Education and Human Services en Cumplimiento parcial de los requisitos para la Obtención del Título de Doctor en Educación.

Salmon, G. (2004) E-actividades. El factor clave para una formación en línea activa. Barcelona: Editorial $\mathrm{UOC}$

Seoane, A y García, F. (s.f.) Introducción al E learning. Recuperado de http://grialdspace.usal.es:443/bitstream/123456789/27/6/introelearning.pdf

Seoane, A.; García, F.; Bosom, A.; Fernández, E. y Hernández, M. J. (2006) Tutoring on-line as quality guarantee on elearning based lifelong learning. Definition, modalities, methodology, competences and skills. Recuperado de http://ceur-ws.org/Vol-186/o5.pdf

UNED (2011). Plan de desarrollo institucional: Para el fortalecimiento de la Educación a Distancia. 20112015. San José, Costa Rica: Vicerrectoría de Planificación.

UNED (2011).Como diseñar y ofertar cursos en línea. Consideraciones generales. San José, Costa Rica: PAL, PACE, UNED.

UNESCO (1998) Declaración mundial sobre la Educación superior en el Siglo XXI: visión y acción. Recuperado de http://www.unesco.org/education/educprog/wche/declaration spa.htm

UNESCO (2009) Conferencia Mundial sobre la Educación Superior - 2009: La nueva dinámica de la educación superior y la investigación para el cambio social y el desarrollo. Recuperado de http://www.unesco.org/education/WCHE2009/comunicado_es.pdf

UNESCO (2009a) ¿Van las TIC hacer la universidad tradicional obsoleta? Recuperado de http://www.unesco.org/new/es/media-services/single-view-tvrelease/news/will_icts_make_the_traditional_university_obsolete-3/ 


\section{Nota acerca de las autoras}

Xinia María Calvo Cruz. Magister en Planificación Curricular. Labora en el Programa de Apoyo Curricular y Evaluación de los Aprendizajes (PACE-UNED), como Asesora Curricular.Teléfono de la oficina: 2527- 2201- celular: 83475887

Correo:xcalvo@uned.ac.cr

Natalia Salas Quirós. Magister en Planificación Curricular. Labora en el Programa de Apoyo Curricular y Evaluación de los Aprendizajes (PACE-UNED), como Asesora Curricular.Teléfono de la oficina: 2527-2747- celular: 8864-8247

Correo:nsalas@uned.ac.cr 